\title{
Magnetic resonance imaging of experimental cerebral oedema
}

\author{
D BARNES, W I McDONALD, P S TOFTS, G JOHNSON, D N LANDON \\ From the Department of Clinical Neurology, Institute of Neurology, Queen Square, London, UK
}

SUMMARY Triethyl tin(TET)-induced cerebral oedema has been studied in cats by magnetic resonance imaging (MRI), and the findings correlated with the histology and fine structure of the cerebrum following perfusion-fixation. MRI is a sensitive technique for detecting cerebral oedema, and the distribution and severity of the changes correlate closely with the morphological abnormalities. The relaxation times, $T_{1}$ and $T_{2}$ increase progressively as the oedema develops, and the proportional increase in $T_{2}$ is approximately twice that in $T_{1}$. Analysis of the magnetisation decay curves reveals slowly-relaxing and rapidly-relaxing components which probably correspond to oedema fluid and intracellular water respectively. The image appearances taken in conjunction with relaxation data provide a basis for determining the nature of the oedema in vivo.

Proton nuclear magnetic resonance (NMR) imaging (MRI) provides a sensitive means of detecting abnormalities in the brain in a variety of diseases. The image appearances, however, lack specificity. For example, the abnormal appearances seen in multiple sclerosis may be indistinguishable from those seen in cerebral vascular disease. ${ }^{1}$

Many pathological processes affecting the central nervous system are associated with alterations in intracellular or extracellular water or both. Since the NMR signal intensity is determined not only by the quantity of water in a tissue but also by its physicochemical environment, it is to be expected that this technique might distinguish between different types of pathological change as a result of differential alterations in proton density and relaxation times, $T_{1}$ and $T_{2}$. The form of the magnetisation decay curve might provice further information about the components of tissue water in the sample.

We have begun a systematic study of the NMR characteristics of abnormal tissue water in brain by studying the oedema produced by intoxication with triethyl tin. This form of oedema affects the cerebral white matter diffusely. ${ }^{2}$ The myelin sheath is disrupted at the intraperiod line and oedema fluid accumulates in the resulting spaces. ${ }^{3}$ In contrast to vaso-

Address for reprint requests: Professor WI McDonald, Institute of Neurology, Queen Square, London WC1N 3BG, UK

Received 4 February 1986. Accepted 24 February 1986 genic oedema the extracellular space is not enlarged, and astrocytic swelling is not conspicuous. The composition of the oedema fluid in the TET lesion also differs from that of vasogenic oedema in that it resembles a plasma ultrafiltrate lacking a significant protein content. ${ }^{4}$ In this paper we describe the evolution of the changes in NMR characteristics of TET-induced cerebral oedema and relate them to the ultrastructural changes.

\section{Methods}

The experiments were performed on 10 adult female cats weighing $2 \cdot 2-3.3 \mathrm{~kg}$ (mean $2.75 \mathrm{~kg}$ ). Oedema was induced by the intraperitoneal injection of one to four doses of triethyl tin sulphate $(1 \mathrm{mg} / \mathrm{kg}$ body weight).

\section{Imaging}

MRI was performed using a Picker International system operating at 0.5 Tesla and a saddle-shaped receiver coil $10 \mathrm{~cm}$ in diameter. The animals were anaesthetised prior to imaging by intraperitoneal injections of pentobarbitone sodium ( $40 \mathrm{mg} / \mathrm{kg}$ body weight).

Total imaging time varied from 3 to 4 hours, during which five to seven inversion recovery (IR) and eight to 10 spin echo (SE) scans were performed.5 Coronal sections were found to be the must useful as they provided the best anatomical detail, and the depth of white matter relative to slice thickness minimised partial volume effects.

Spin echo sequences consist of an initial $90^{\circ}$ radiofrequency (RF) pulse, followed after an interval by a $180^{\circ}$ pulse. This second pulse produces a spin echo signal at an interval (the echo time, $T_{e}$ ) after the initial $90^{\circ}$ pulse. If the 
echo time is increased, the magnitude of the signal from a given tissue decreases at a rate determined by its transverse relaxation time, $T_{2}$. Thus tissues with very long $T_{2}$ values remain visible at the highest echo times. In the later experiments the basic spin echo sequences were modified by the addition of a second $90^{\circ}$ pulse which saturates the magnetisation after the echo (the "SAFE" pulse-PS Tofts, unpublished). By allowing a constant recovery period after the SAFE pulse before beginning the next sequence, a linear dependence between $T_{e}$ and signal intensity was achieved. This would otherwise not be the case for spin echo sequences which employed very long echo times since a significant dependence on $T_{1}$ would be introduced. SAFE pulses minimise this effect.

Images produced by inversion recovery sequences are more dependent on the longitudinal relaxation time $T_{1}$, and consist of an initial $180^{\circ}$ (inversion) pulse followed by a $90^{\circ}$ pulse and a further $180^{\circ}$ pulse which produces the signal echo. During the interval between the first two pulses (the inversion time, $T_{i}$ ) the magnetisation returns towards its equilibrium state at a rate dependent on $T_{1}$. By varying $T_{i}$, differences in $T_{1}$ of tissues can be highlighted. In the present experiments values of $T_{i}$ up to $700 \mathrm{~ms}$ and $T_{e}$ up to $800 \mathrm{~ms}$ were used.

All scans employed a repetition time of 2 seconds, and the reconstruction method used was two-dimensional Fourier transform (2DFT) imaging. The pixel dimensions were 0.6 $\times 1.2 \mathrm{~mm}$, and the slice width $5 \mathrm{~mm}$.

A bottle of manganese chloride $\left(\mathrm{T}_{1}=640 \mathrm{~ms}, \mathrm{~T}_{2}=\right.$ $154 \mathrm{~ms}$ ) was included in the imaging field to act as a reference, which enabled us to ensure that the images and calculated relaxation times remained consistent from one occasion to another.

The calculated images were produced by computer algorithms: the $T_{1}$ image from an $S E\left(T_{e}=40 \mathrm{~ms}\right)$ and an IR ( $T_{i}$ $=700 \mathrm{~ms})$, the $T_{2}$ from two $S E\left(T_{e}=40\right.$ and $\left.120 \mathrm{~ms}\right)$ scans.

Magnetisation decay curves were constructed by plotting signal intensity against echo time on a log-linear scale. A computed least-squares fitting procedure was applied to derive the best monoexponential, biexponential and triexponential fits. An F-test was applied to determine the best fit.

\section{Determination of water content}

Three animals were killed immediately after the final imaging session following 4 days of TET by an overdose of anaesthetic following which the brains were rapidly removed and sectioned, and samples of white and grey matter taken for wet-dry weight determination of water content. These samples were dried at $60^{\circ} \mathrm{C}$ until their weights were stable for 48 hours.

\section{Histology}

Two animals were killed at two and four days by perfusionfixation through the ascending aorta via the left ventricle using $3 \%$ glutaraldehyde in $0.1 \mathrm{M}$ cacodylate buffer at pH 7.4. $1500 \mathrm{mls}$ of fixative were perfused over 15 minutes at an effective pressure of $180 \mathrm{mmHg}$. The brains were removed after a further 48 hours in fixative and tissue samples were post-fixed in $1 \%$ osmium tetroxide solution, dehydrated in graded ethanol solutions, and embedded in epoxy resin for microscopy. Semi-thin sections $(0.5 \mu \mathrm{m})$ were stained with toluidine blue for light microscopy, and ultra-thin sections $(60 \mathrm{~nm})$ with Reynold's lead citrate and methanolic uranyl acetate for transmission electron microscopy.

One animal was perfused with formol-saline for paraffin preparations for light microscopy.

\section{Results}

The susceptibility of individual animals to TET varied, and the dosage regime was adjusted so as to produce comparable abnormalities on the NMR images.

\section{Microscopic appearances}

The predominant abnormality observed by light microscopy was extensive vacuolar change in the cerebral white matter (fig 1).

The most obvious difference between the appearances at 2 and 4 days was the size rather than the number of vacuoles. The cortex was not completely spared; a similar vacuolar appearance was occasionally seen in the layers adjacent to white matter, although the neurons themselves appeared normal. A further abnormality of the grey matter was the occasional finding of enlargement of the Virchow-Robin spaces around the penetrating vessels.

Oedema was found throughout the cerebral hemispheres, but was not uniformly distributed, the gyral white matter, that adjacent to cortex and basal ganglia, and the corpus callosum being most severely affected.

The predominant ultrastructural abnormality was severe disruption of the myelin sheaths as shown in figure 2. The myelin lamellae were separated at the intraperiod line. Individual sheaths showed considerable variation in the extent to which they were disrupted, and grossly vacuolated examples could be found adjacent to apparently normal ones. The

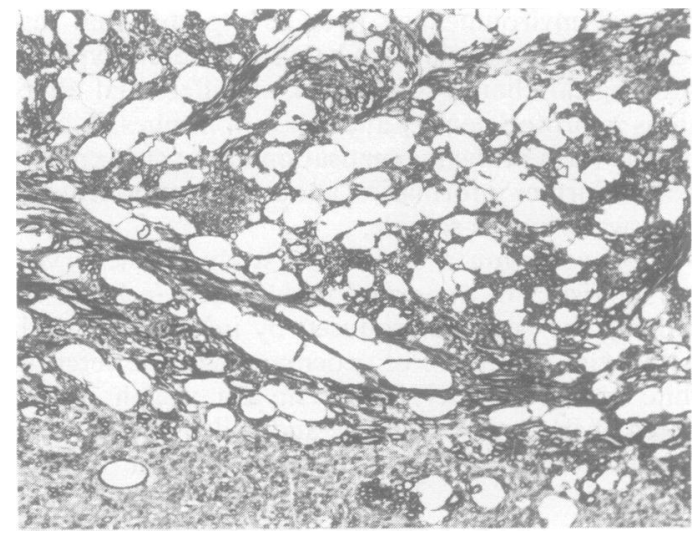

Fig 1 Light microscopic appearance of the vacuolar change seen in the white matter in TET oedema (Toluidine blue, $\times 280$ ). 


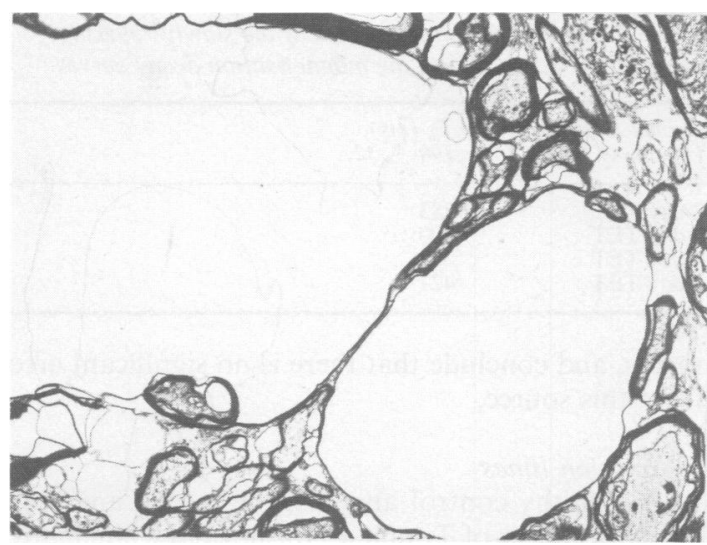

Fig 2 Electron micrograph of large intramyelinic vacuoles. They are devoid of electron-dense material $(\times 10,800)$.

majority of sheaths were affected to some extent in both early and late lesions. The vacuoles always appeared to be clear, and devoid of electron-dense material. The extracellular space was not enlarged and there was little swelling of astrocytic processes.

The main difference between early and late changes (also seen by light microscopy) was the size of the vacuoles which increased progressively with time, rarely exceeding $12 \mu \mathrm{m}$ in diameter at 2 days, but frequently exceeding $15 \mu \mathrm{m}$ at 4 days.

\section{Scan appearances}

The normal pattern of white matter in coronal section and a corresponding spin echo image from a control animal are shown in fig 3 . Figure 4 shows images produced by two different spin echo sequences in which the widespread changes caused by the TET can be seen. In keeping with the histological changes, the degree of signal enhancement was generally greatest in the gyri and in the white matter of the internal capsule adjacent to the basal ganglia. The corpus callosum could not be assessed owing to its small size and proximity to the high signal generated by CSF within the ventricles. Unlike normal white matter from which no signal was seen beyond echo times of about $400 \mathrm{~ms}$, signal from oedematous tissue was still present at echo times up to $800 \mathrm{~ms}$. The grey matter was of normal appearance in all scans.

\section{Magnetisation decay}

A signal- $T_{e}$ plot was constructed on a log-linear scale in order to determine the characteristics of the magnetisation decay which would be expected to provide information about the magnitude and relaxation rate of the major tissue water components. Prior to the use of the SAFE pulses normal white matter showed linear magnetisation decay characteristics. In the later experiments, however, when SAFE pulses were used, samples of normal white matter consistently showed biexponential behaviour. In oedematous white matter the magnetisation decay was always observed to be non-linear, but in no experiment did the addition of a third exponential function significantly improve the fit, and all non-linear decays were therefore treated as biexponential.

The characteristics of the signal decay at different times are shown in fig 5 . In the 2 day lesions the slowly-relaxing component was found to constitute approximately $20 \%$ of the signal, whereas by 4 days this had risen to $25-30 \%$. The $\mathrm{T}_{2}$ value of this com-
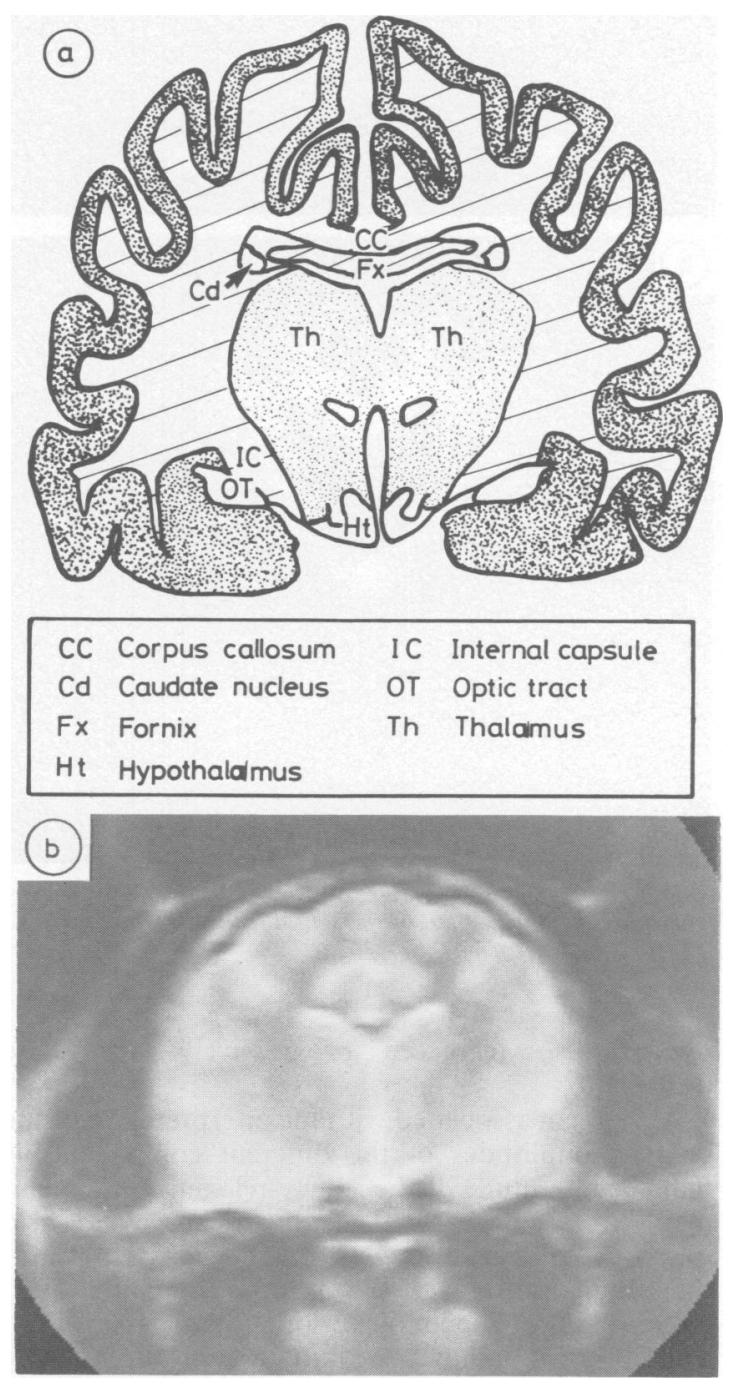

Fig 3 (a) Schematic coronal section through the cat brain at the level of the thalamus. (b) Corresponding magnetic resonance image of healthy animal $\left(T_{\mathrm{c}}=120 \mathrm{~ms}\right)$. 

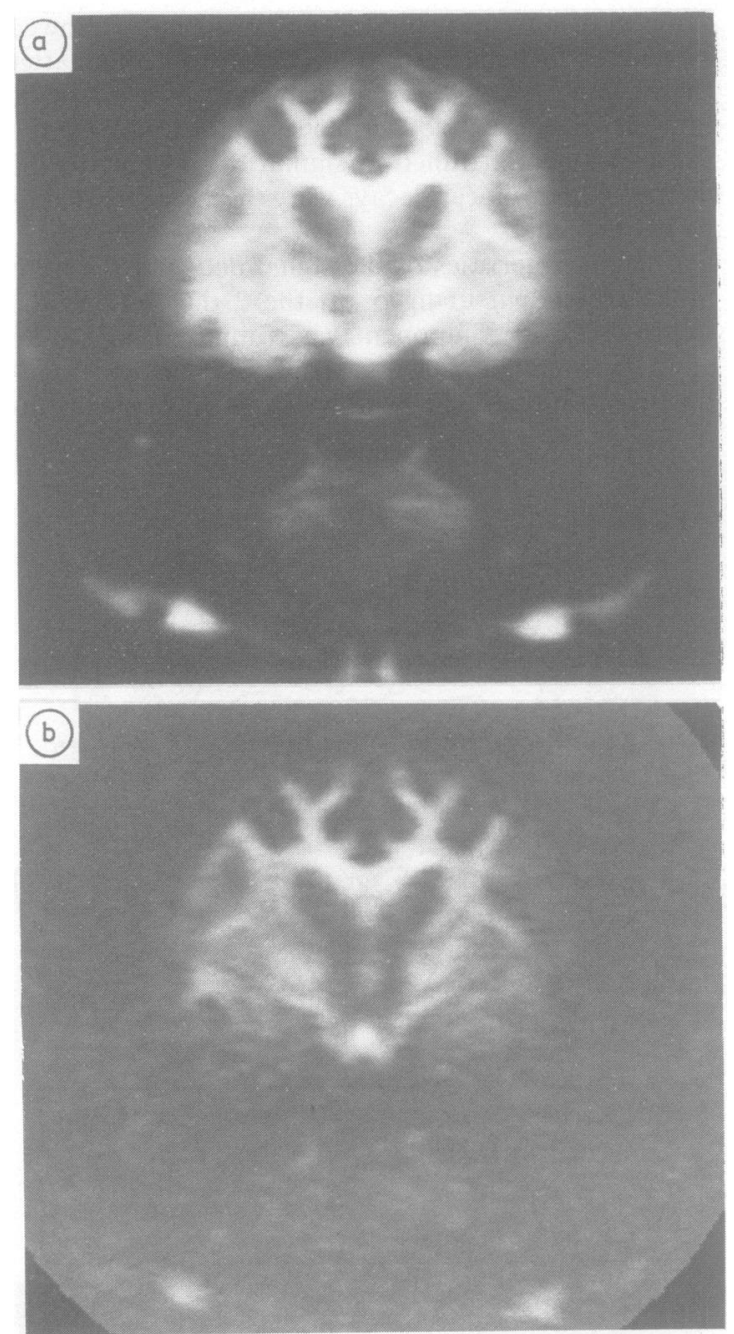

Fig 4 Images in the coronal plane demonstrating generalised enhancement of the signal from the white matter. (a) $T_{\mathrm{e}}=160 \mathrm{~ms}$. (b) $T_{\mathrm{e}}=320 \mathrm{~ms}$.

ponent also increased progressively with time (table 1).

A problem associated with the interpretation of the relative amplitudes of the different components is that the amplitude of the slowly-relaxing component may be falsely lowered if the $T_{1}$ decay is also biexponential. This results from partial saturation of the magnetisation at the beginning of the next sequence due to incomplete longitudinal relaxation. This effect would lower the initial signal amplitude by about $35 \%$ if $\mathrm{T}_{1}$ were 2 seconds as previously reported. ${ }^{11}$ We have not, however, been able to demonstrate a $T_{1}$ biexponentiality in any of the experi-
Table 1 The $T_{2}$ relaxation times of the slowly-relaxing components derived from the magnetisation decay curves.

\begin{tabular}{ll}
\hline $\begin{array}{l}\text { Animal } \\
(n=6)\end{array}$ & $T_{2}(\mathrm{~ms})$ \\
& $190 \pm 32$ \\
\hline 2 day TET & 283 \\
3 day TET & 300 \\
4 day TET & 312 \\
4 day TET & 427 \\
\hline
\end{tabular}

ments, and conclude that there is no significant error from this source.

\section{Relaxation times}

In 36 healthy control animals, the means and standard deviations of $T_{1}$ and $T_{2}$ for normal white matter were $476 \pm 10 \mathrm{~ms}$ and $81 \pm 4 \mathrm{~ms}$ respectively, and the values for each animal with cerebral oedema are listed in table 2. It is more useful to compare percentage changes than absolute values since this facilitates comparison between animals with different values for normal tissue. In all cases the increase in $T_{2}$ was approximately twice that in $T_{1}$. This is shown as a

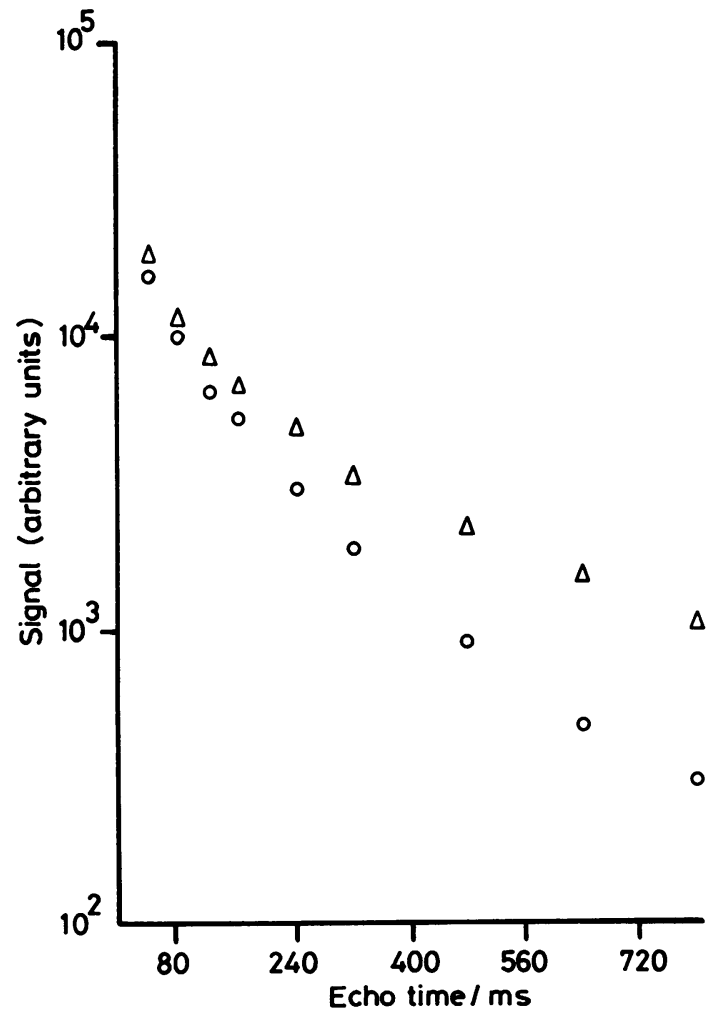

Fig 5 Magnetisation decay curves showing the non-linear decay characteristics seen at 2 days $(o)$ and 4 days $(\Delta)$ after the first injection. 


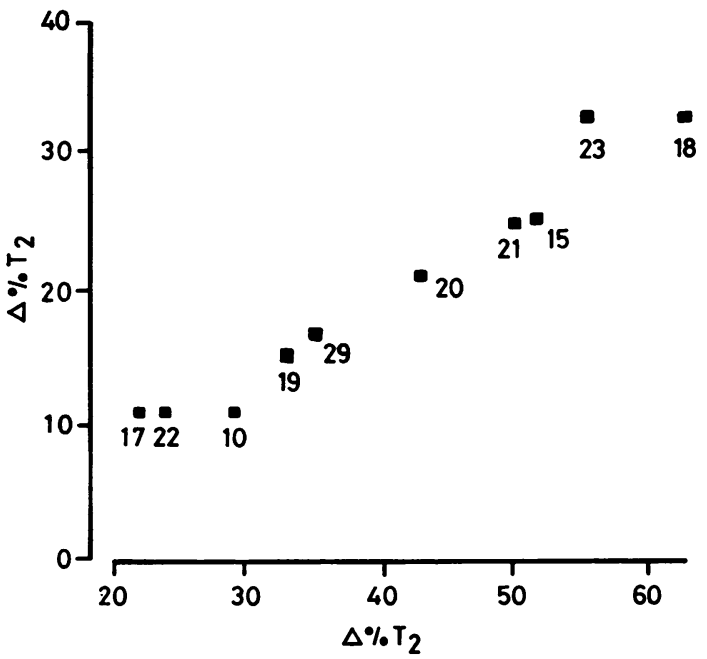

Fig 6 A scatter plot showing the percentage increase in the relaxation times of the oedematous white matter in each experimental animal (the numbers refer to individual animals, cf. table 2.)

scatter plot in fig 6, in which all degrees of severity of oedema result in an approximately linear relationship between the two parameters regardless of the characteristics of the signal decay.

\section{Water content}

The increase in water content is a measure of the severity of the oedema. The water content of normal white matter, as determined by wet-dry weight estimations, was derived from four control animals and was found to be $65.9 \pm 2 \%(n=4)$. The water content was increased to $72 \cdot 2 \pm 2 \cdot 9 \%(n=3)$ in the most severe lesions representing an increase, calculated to dry weight, of about $35 \%$.

\section{Discussion}

Our morphological observations correspond closely

Table 2 The relaxation times of the oedematous white matter in each experimental animal

\begin{tabular}{lll}
\hline Animal number & $T_{1}(\mathrm{~ms})$ & $T_{2}(\mathrm{~ms})$ \\
\hline 10 & 540 & 110 \\
15 & 612 & 131 \\
17 & 510 & 97 \\
18 & 640 & 132 \\
19 & 550 & 110 \\
20 & 580 & 117 \\
21 & 605 & 121 \\
22 & 520 & 99 \\
23 & 631 & 125 \\
29 & 563 & 114 \\
\hline
\end{tabular}

to those of previous reports; ${ }^{367}$ there were generalised changes throughout the cerebral hemispheres which consisted of progressive accumulation of oedema fluid within vacuoles following the separation of myelin lamellae at the intraperiod line. The absence of electron-dense material from the vacuoles was in keeping with previous reports that the oedema fluid is chemically similar to a plasma ultrafiltrate, lacking a significant protein content. ${ }^{47}$

The MRI appearance of the oedematous white matter was abnormal from an early stage and became progressively more so as the oedema developed. The severity of the oedema in different areas of white matter corresponded closely to the distribution of changes seen on the NMR images.

The relaxation times, $T_{1}$ and $T_{2}$ lengthened as the water content of the oedematous white matter increased, although at all stages the percentage increase in $T_{2}$ was approximately twice that in $T_{1}$. Consequently, the more $T_{2}$-dependent spin echo sequences were more useful in demonstrating the abnormalities in these experiments.

$T_{1}$ and $T_{2}$ relaxation are both dependent upon interactions with fluctuating magnetic fields and represent related physical processes. ${ }^{8}$ It is not surprising, therefore, that there is a tendency for changes in these parameters to follow a similar course. There are differences in their physical significance, however, which may provide an explanation for the finding of a greater proportional increase in $T_{2}$ than $T_{1}$ in this type of oedema. The $T_{1}$ relaxation process depends mainly upon components of molecular motion with correlation times corresponding to the resonance frequency (in the $\mathrm{MHz}$ range), whereas $\mathrm{T}_{2}$ relaxation is most sensitive to the static components. ${ }^{9}$ In bulk water, $T_{1}=T_{2}$, whereas in tissues the presence of macromolecular structures, which exert motional restriction upon water molecules, enhances both relaxation processes, and modifies $T_{1}$ and $T_{2}$ in such a way that the $T_{1}$ of normal white matter is about six times as long as the $T_{2}$. In TET-induced oedema, the increase in bulk water would therefore be expected not only to prolong both the observed $T_{1}$ and $T_{2}$, but also to produce a greater proportional increase in $T_{2}$, which was in fact what was found.

Although the $T_{1}$ and $T_{2}$ values reliably reflected the severity of the oedema, the absolute values may be misleading because the algorithm by which they were calculated uses a two-point method which is therefore unable to detect non-linear magnetisation decays. More accurate information about the relaxation behaviour, and further information about the size and state of tissue water compartments can be obtained by measuring the magnetisation decay characteristics of the tissue.

The magnetisation decay characteristics of normal 
white matter were non-linear in the experiments in which the SAFE pulses were used. Oedematous white matter, however, showed non-linear decay in all experiments, with or without the SAFE pulses. Such non-linearity would be expected if there was more than one component corresponding to proton populations with different relaxation properties at the tissue level. ${ }^{8}$ This would be observed (1) when the different compartments were separated by a barrier to diffusion such as the cell membrane which separates intracellular and extracellular water; (2) when the microscopic heterogeneity of a tissue is such that protons cannot diffuse sufficiently rapidly between areas with different physical properties; or (3) when bound and unbound water cannot exchange rapidly relative to their relaxation times.

Before the introduction of the SAFE pulses we had been unable to demonstrate a significant biexponentiality in normal white matter. Previous reports are inconclusive on this point. ${ }^{1012}$ The extracellular space in white matter is thought to be about $15 \%$ by volume. ${ }^{13} 14$ If this is true, and if intracellular and extracellular water have sufficiently different relaxation properties, then a non-linear decay curve would be expected. Prior to the use of the SAFE pulses this had been concealed by a tendency for the signal amplitude to be falsely lowered at very long echo times.

The oedematous tissue consistently yielded two components. The $T_{2}$ of the more rapidly relaxing component showed little variation and lay in the range of 50-67 ms. The relative amplitudes of the two components varied with the severity of the oedema; the slowly-relaxing component becoming proportionally larger as the oedema progressed. Furthermore, the $T_{2}$ of this component was found to be greatest in the most severe lesions. It is probable that the long- $T_{2}$ component represents oedema fluid since the time course of the increase in its relative amplitude correlates with the progressive enlargement of the vacuoles, and its $T_{2}$ value is consistent with the chemical nature of the oedema. The large constant- $T_{2}$ component probably represents intracellular water which is unaffected by TET.

We have treated non-linear magnetisation decay as biexponential, and have been unable to improve the fit by the addition of a third exponential function. It is likely that further tissue water compartments do contribute to the signal, but that their rate of relaxation is too great to be detected by our technique. ${ }^{15}$

Our in vivo findings are in keeping with those derived from in vitro samples of brain tissue from rats intoxicated with TET in which, however, morphological correlations were not described. ${ }^{1011}$ The magnetisation decay of normal white matter was monoexponential in those experiments, but after 48 hours of TET administration, biexponentiality was observed. It is uncertain to what extent these findings can be extrapolated to the in vivo state since expansion of the intracellular space after cell death, the possible derangement of tissue barriers, and the effects of tissue handling could combine to alter the NMR characteristics of the tissue in an unpredictable fashion.

We have shown that it is possible to obtain accurate and precise quantitative NMR data about one form of pathological alteration in tissue water, and that these data are in keeping with the known ultrastructural and chemical features of the oedema. The next step will be to determine whether lesions with different morphological and chemical features have specifically different NMR characteristics. If this is found to be the case, the usefulness of MRI might be extended to the determination of more specific pathological and chemical information about changes in the human brain during life.

Our present observations may have some relevance to the clinical problem of Reye's syndrome. The cerebral oedema associated with this acute encephalopathy of childhood is morphologically very similar to that induced by TET. ${ }^{16}$ There are differences, however, such as the marked astrocytic swelling seen in Reye's syndrome, but the absence of conspicuous inflammatory changes and the sequestration of oedema fluid within intramyelinic vacuoles suggest that their NMR characteristics might be similar. MRI, which has so far proved to be without known biological hazard, could be of value in monitoring the progress of the disease and its response to treatment.

We are grateful to Mr Alan Crockard and the Cerebral Oedema Research Group for carrying out the measurements of tissue water content, and to Miss Jenny Small for expert technical assistance with the morphological studies and illustrations.

The work was carried out on the MRI facility provided by the Multiple Sclerosis Society, and was supported by a grant from the MRC.

\section{References}

1 Ormerod IEC, Du Boulay GH, McDonald WI. Imaging and Multiple Sclerosis. In: McDonald WI, Silverberg DH, eds. Multiple Sclerosis. London: Butterworths, 1986.

2 Magee PN, Stoner HB, Barnes JM. The experimental production of oedema in the central nervous system of the rat by triethyl tin compounds. J Pathol Bact 1957;73:107-23.

3 Lee JC, Bakay L. Ultrastructural changes in the edematous central nervous system. Arch Neurol 
1965;13:48-58.

4 Bakay L. Morphological and chemical studies in cerebral oedema: triethyl-tin-induced oedema. J Neurol Sci 1965;2:52-67.

5 Young IR, Bailes DR, Burl M, et al. Initial clinical evaluation of a whole body nuclear magnetic resonance (NMR) tomograph. J Comput Assist Tomogr 1982;6(1):1-18.

6 Hirano A, Zimmerman HM, Levine S. Intramyelinic and extracellular spaces in triethyl tin intoxication. $J \mathrm{Neu}$ ropathol Exp Neurol 1968;27:571-80.

7 Aleu F, Katzman R, Terry RD. Fine structure and electrolyte analysis of cerebral oedema induced by alkyl tin intoxication. J Neuropathol Exp Neurol 1963; 22:403-12.

8 Mathur-De Vre R. Biomedical implications of the relaxation behaviour of water related to NMR imaging. $\mathrm{Br}$ J Radiol 1984;57:955-76.

9 Berendsen HJC. Specific interactions of water with biopolymers. In: Felix Franks, ed. Water: $A$ Comprehensive Treatise. New York and London: Plenum Press, 1975.

10 Naruse S, Horikawa Y, Tanaka C, et al. Proton nuclear magnetic resonance studies on brain edema. $J$ Neu- rosurg 1982;56:747-52.

11 Go KG, Edzes HT. Water in brain edema. Arch Neurol 1975;32:462-6.

12 Bakay L, Kurland RJ, Parrish RG, et al. Nuclear magnetic resonance studies in normal and edematous brain tissue. Exp Brain Res 1975;23:241-8.

13 Rees S, Cragg BG, Everitt AV. Comparison of extracellular space in the mature and ageing rat brain using a new technique. J Neurol Sci 1982;53:347-57.

14 Van Harreveld A. The extracellular space in the vertebrate central nervous system. In: Bourne GH, ed. The Structure and Function of Nervous Tissue. London: Academic Press, 1972.

15 Foster KR, Resing HA, Garroway AN. Bounds on "Bound Water": Transverse nuclear magnetic resonance relaxation in barnacle muscle. Science 1976; 194:324-6.

16 Partin JC, Partin JS, Schubert WK, McLaurin RL. Brain ultrastructure in Reye's syndrome. $J$ Neuropathol Exp Neurol 1975;34:425-44.

17 Budinger TF. Nuclear magnetic resonance (NMR): In vivo studies. Known thresholds for health effects. $J$ Comput Assist Tomogr 1981;5:800-11. 
bilitation scene in Denmark and in the United Kingdom, and his views are at odds with those reported in the book. Christensen and Danielson review the scene in Denmark, but restrict their attention largely to a recent Luria-driven approach, and only pay brief lipservice to the important work of Thomsen and others in Hornbaeck Hospital, a hospital supplying rehabilitation services for the past 25 years. The British scene is described by Wilson, and is disturbingly selective. Nowhere is there any mention of the important work on neurorehabilitation carried out under the aegis of the Armed Forces, and the important work of Eames and his colleagues in behavioural approaches appears to go without mention.

In conclusion, this book is good in parts. The second part is by far the most valuable, but overall it is difficult to see what unique contribution the book makes to the growing literature on neuropsychology and rehabilitation.

NEIL BROOKS

The Bramwells of Edinburgh. By Bryan Ashworth. (Pp 96; £13.00.) Edinburgh: The Royal College of Physicians, 1986.

The coat of arms of the Bramwell family includes the motto "Like Begets Like" and Bryan Ashworth worthily records the truth of that sentiment in this account of the Bramwell dynasty of doctors. Their forebear was the Reverend William Bramwell (1759-1818) a fanatical evangelist whose influence on General Booth is evidenced in his naming his son after him. Dr William Bramwell (1792-1854) in turn sent two sons into medicine. Dr John Byrom (1823-1882) had thirteen children among whom Byrom Bramwell (1847-1931) was educated at Cheltenham College and Edinburgh Medical School. He became House Surgeon to James Spence but refused a post with Laycock to return to rescue his father's general practice in North Shields. He moved via Newcastle in 1874 to the new Royal Infirmary in Edinburgh in 1879. He confined his work entirely to consulting practice. Kinnier Wilson, his one time House Physician dedicated his book to him. He was the leading physician in Scotland though he was not elected to a chair. He was author of many books and papers particularly in neurology and was President of the Royal College of Physicians of Edinburgh (1910-1912) and in 1924 accepted a knighthood. Two of his sons qualified in medicine. Edwin Bramwell (1873-1952) was appointed to the Moncrieff-Arnott Chair of Clinical Medicine and John Crighton Bramwell (1889-1976) became Professor of Cardiology in Manchester and Physician to the Manchester Royal Infirmary. Edwin's disappointing career at Cheltenham led at least one master to despair of him. He resolved nevertheless to take up medicine. (How sad that cognitive rather than genetic traits are now the basis of selection). After a modest undergraduate career, he embarked upon a European tour of self-education which launched him on the stunning orbit which culminated in the Presidency of the Royal College of Physicians of Edinburgh in 1933. John Crighton went from Cheltenham to Trinity, Cambridge where he took a first before reading Medicine in Manchester where, apart from his Rockefeller Scholarship, he remained.

Bryan Ashworth illuminates his text with interesting sidelights on eminent personalities as they interact with the Bramwell saga and with some fascinating appendices. This is a good read for anyone wanting to see the evolution from Victorian to modern medical training and practice. Medical students might read it with profit.

DC TAYLOR

The Chromosomes and Their Disorders 4th ed. (An Introduction for Clinicians.) By GH Valentine. (Pp 223; £17.95.) London: William Heinemann, 1986.

This book is written by the type of clinician I would recommend to all parents who have had the misfortune of having a child with a chromosomal abnormality. In the absence of the clinician (he lives in Canada) I would recommend that we read his book. Parents would then get an explanation about a complex chromosomal re-arrangement in a way that they would understand and they would feel at the end of the consultation that, at least in part, "nature's imperfect design" might not altogether alienate them from the rest of society. The book is totally unfussy about science and the facts are accurate although unreferenced. Indeed the author makes no bones about saying that references would be out of place in this sort of book.

It is a book I would recommend to clinicians in a hurry to understand cytogenetics and to medical students if they had time to read books, to intelligent parents seeking an understandable text (and there are a number of simple explanations worthy of being tucked away in the mind to be used at the next out-patients).

The author admits that he finds dysmorphology difficult and when he strays into that subject there are errors like Noonan syndrome being sometimes autosomal recessive and that there is no prenatal diagnosis for Meckel syndrome, but this is not the sort of book I would consult for those facts. I would highly recommend this fourth edition and wish Dr Valentine to write a fifth edition - even in retirement. MICHAEL BARAITSER

\section{Correction}

Barnes et al Magnetic resonance imaging of experimental cerebral oedema ( $J$ Neurol Neurosurg Psychiatry 1986;49:1341-7.) In figure 6 the ordinate should have been labelled $\Delta \% \mathrm{~T}_{1}$. 